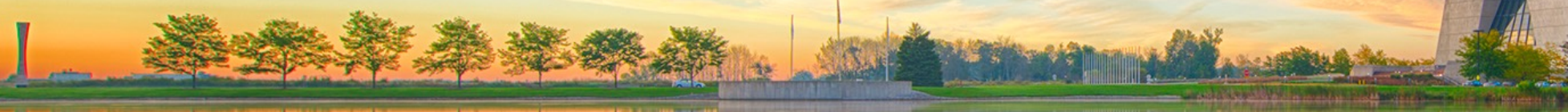
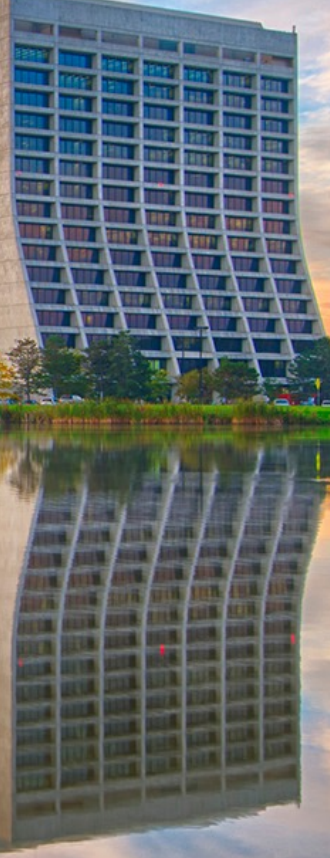

\title{
Accelerator Integration in CMS
}

Dr Christopher Jones

HOW 2019

19 March 2019

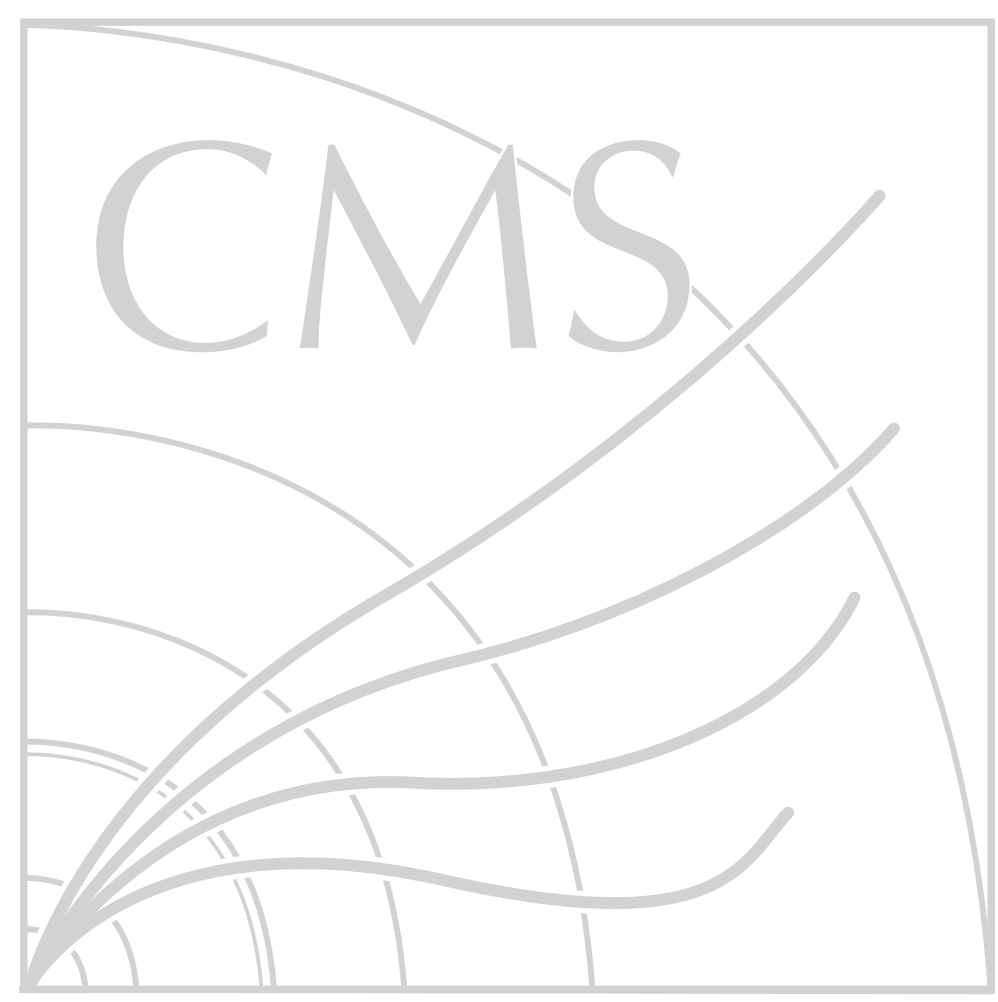




\section{Considerations for Heterogeneous Computing}

\section{Scheduling CPU \& Accelerator Algorithms}

Configuring Heterogeneous Jobs 


\section{Scheduling CPU \& Accelerator Algorithms}




\section{Concurrent CPU/Non-CPU Processing}

CMS data processing framework uses a mechanism to interact effectively with non-cpu resources

Non-CPU algorithms are divided into 3 phases

CPU stage which acquires data and transfers to non-CPU resource

Non-CPU algorithm is then run

When finished, a publish step is run on the CPU to move data back to CPU memory

While non-CPU algorithm runs, the CPU is available for other algorithms 


\section{Setup}

TBB controls running modules

Can have concurrent processing of multiple events

Have separate helper thread to control GPU

Waits until enough work has been buffered before running GPU kernel
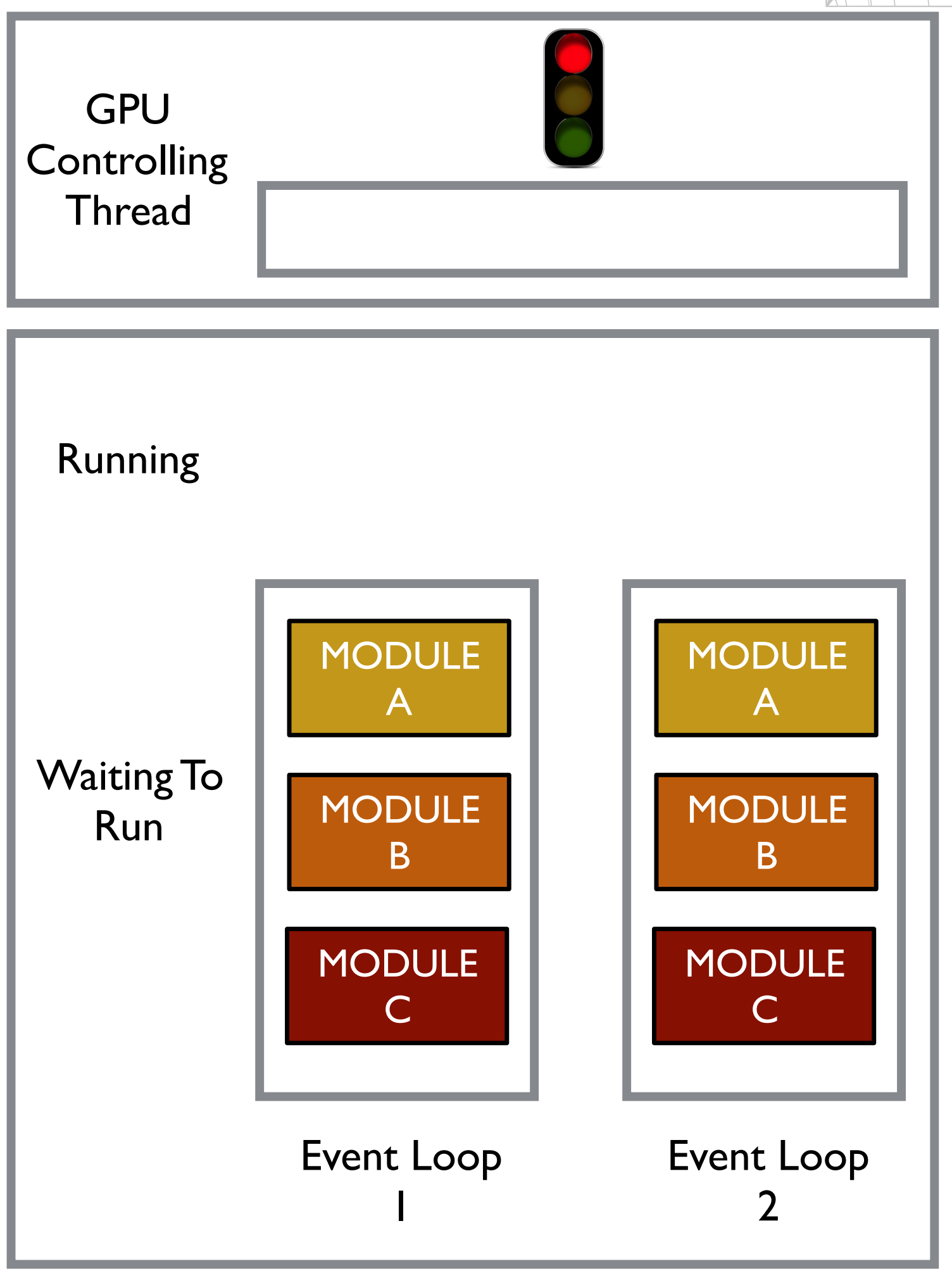


\section{Acquire}

Module acquires method called Used to pull data from Event

Copies data to buffer

Includes a callback to start next phase of module running

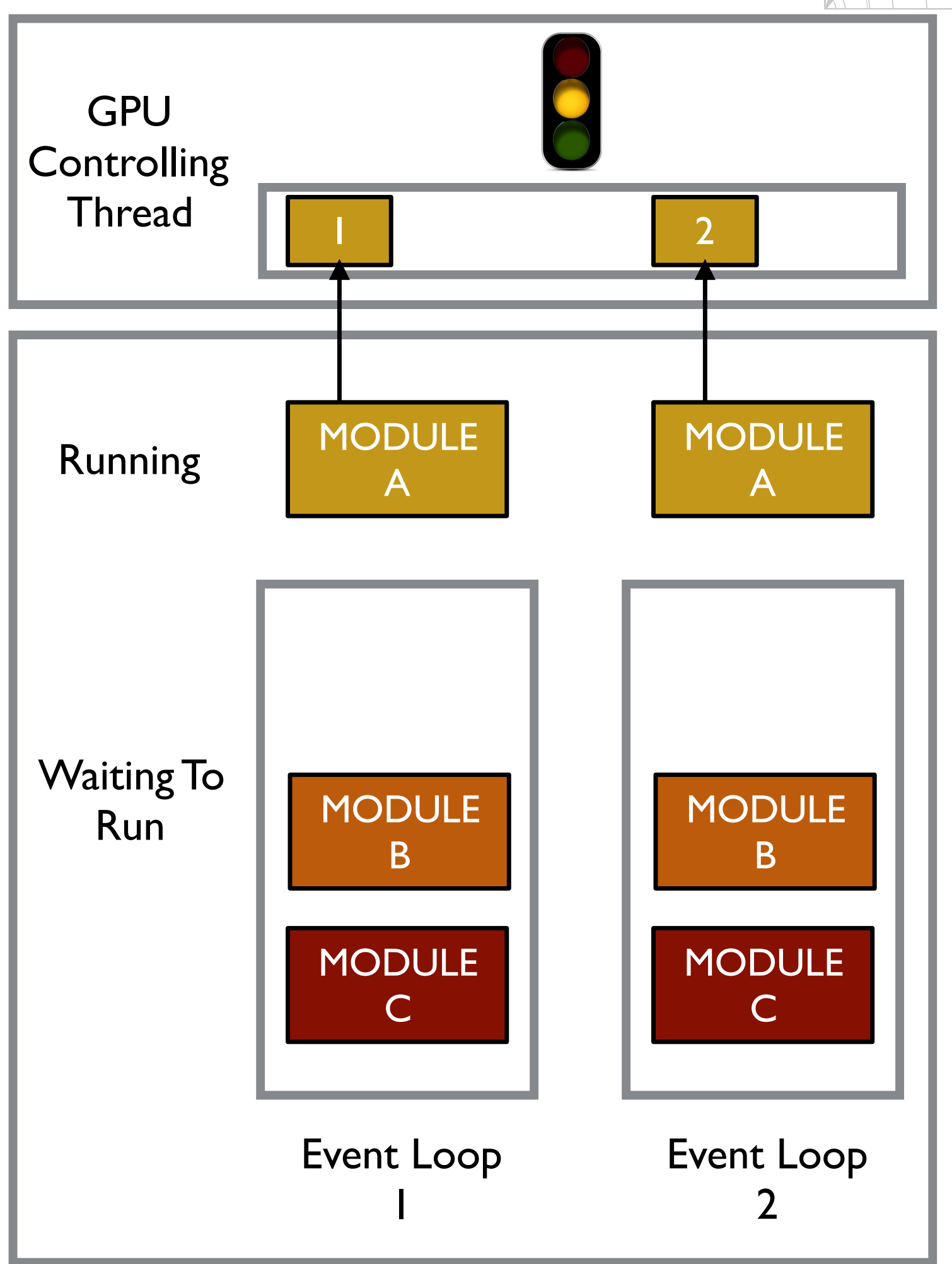

艺 Fermilab 


\section{External Work Starts}

\section{GPU kernel is run}

Data pulled from buffer

Next waiting module can run
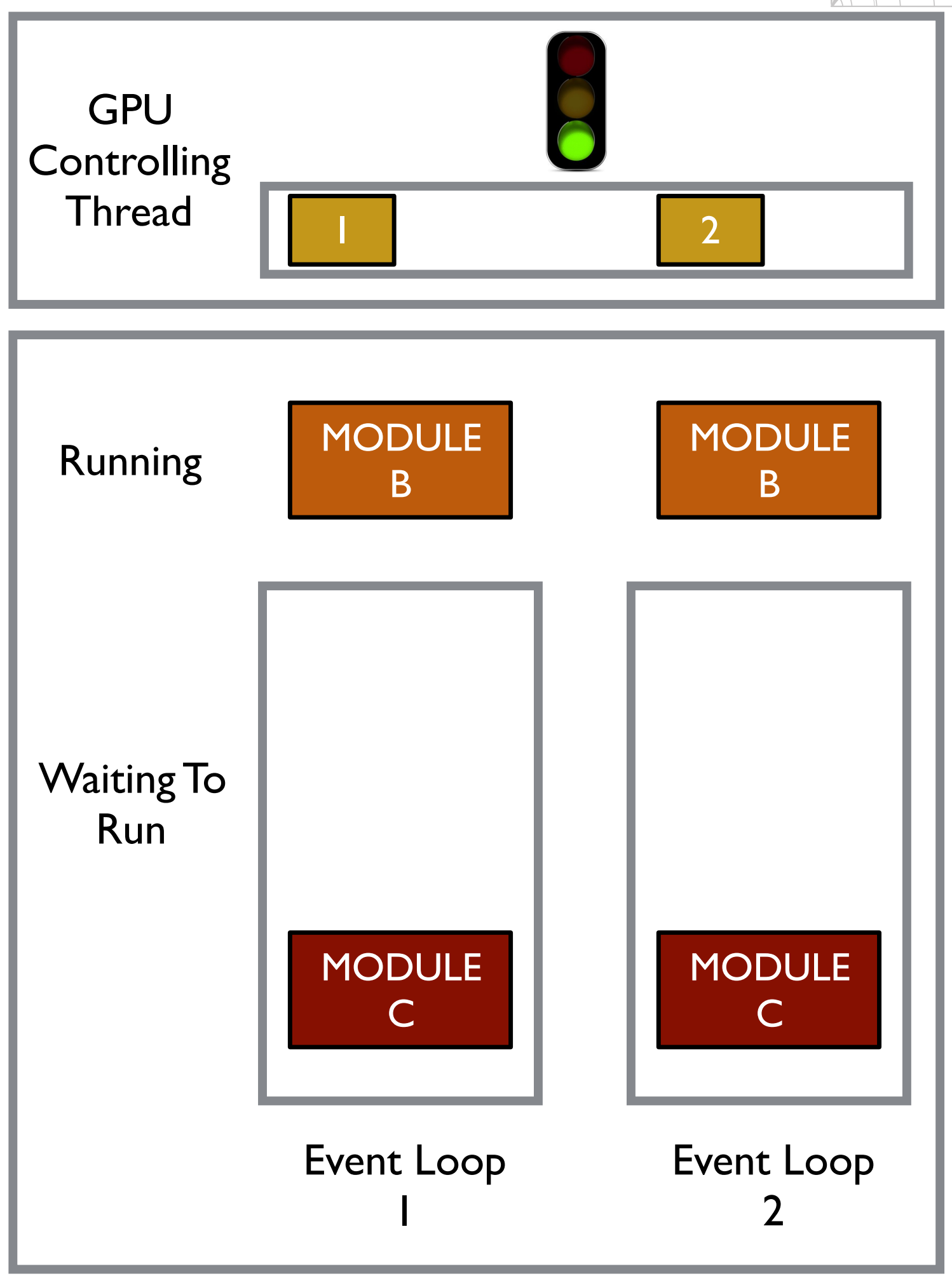

\# Fermilab 


\section{External Work Finishes}

GPU results are copied to buffer

Callback puts Module back into waiting queue

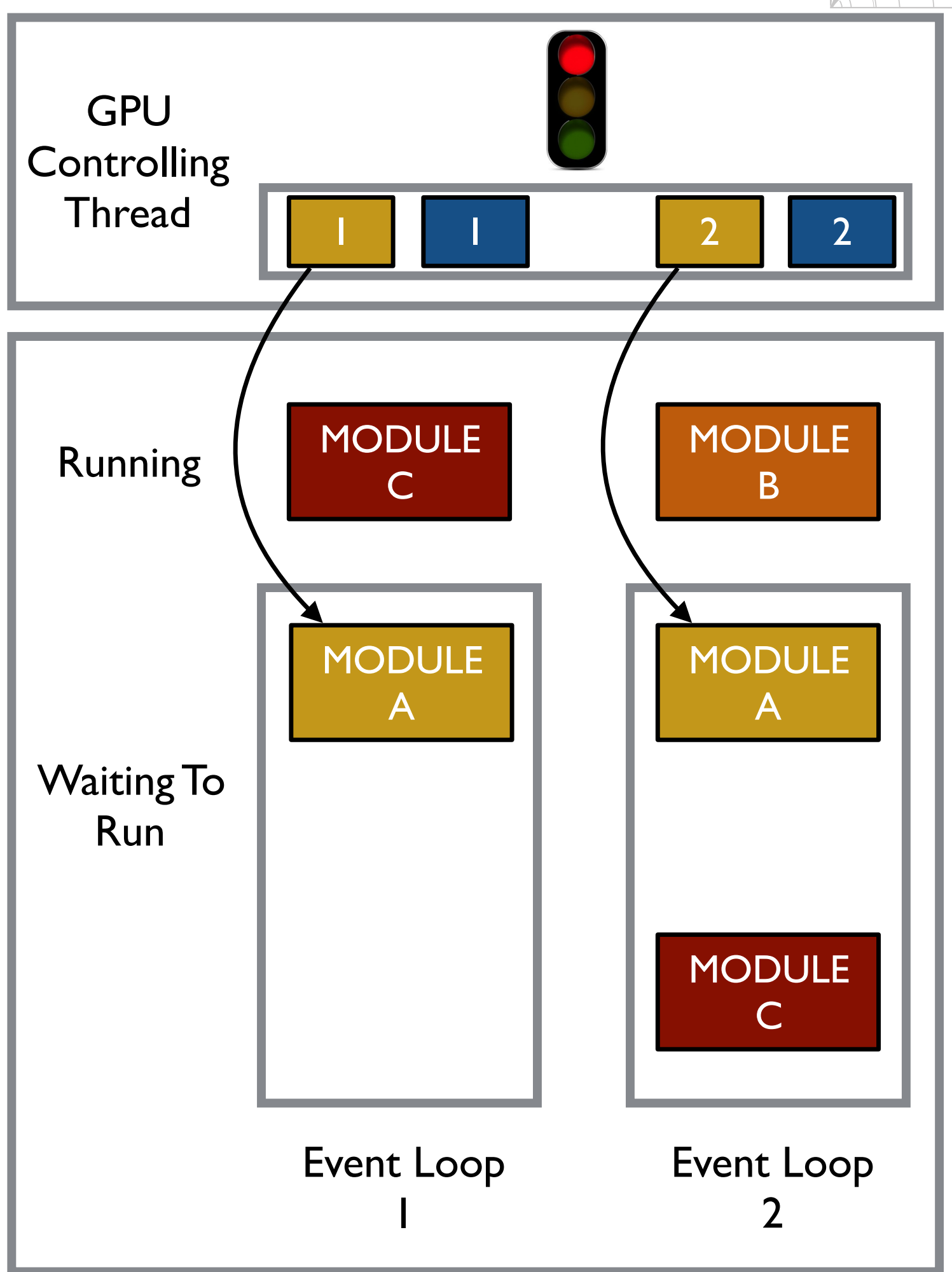

ฟैFermilab 


\section{Produce}

Produce method of module is called

Pull data from buffer

Data used to create objects to put into Event

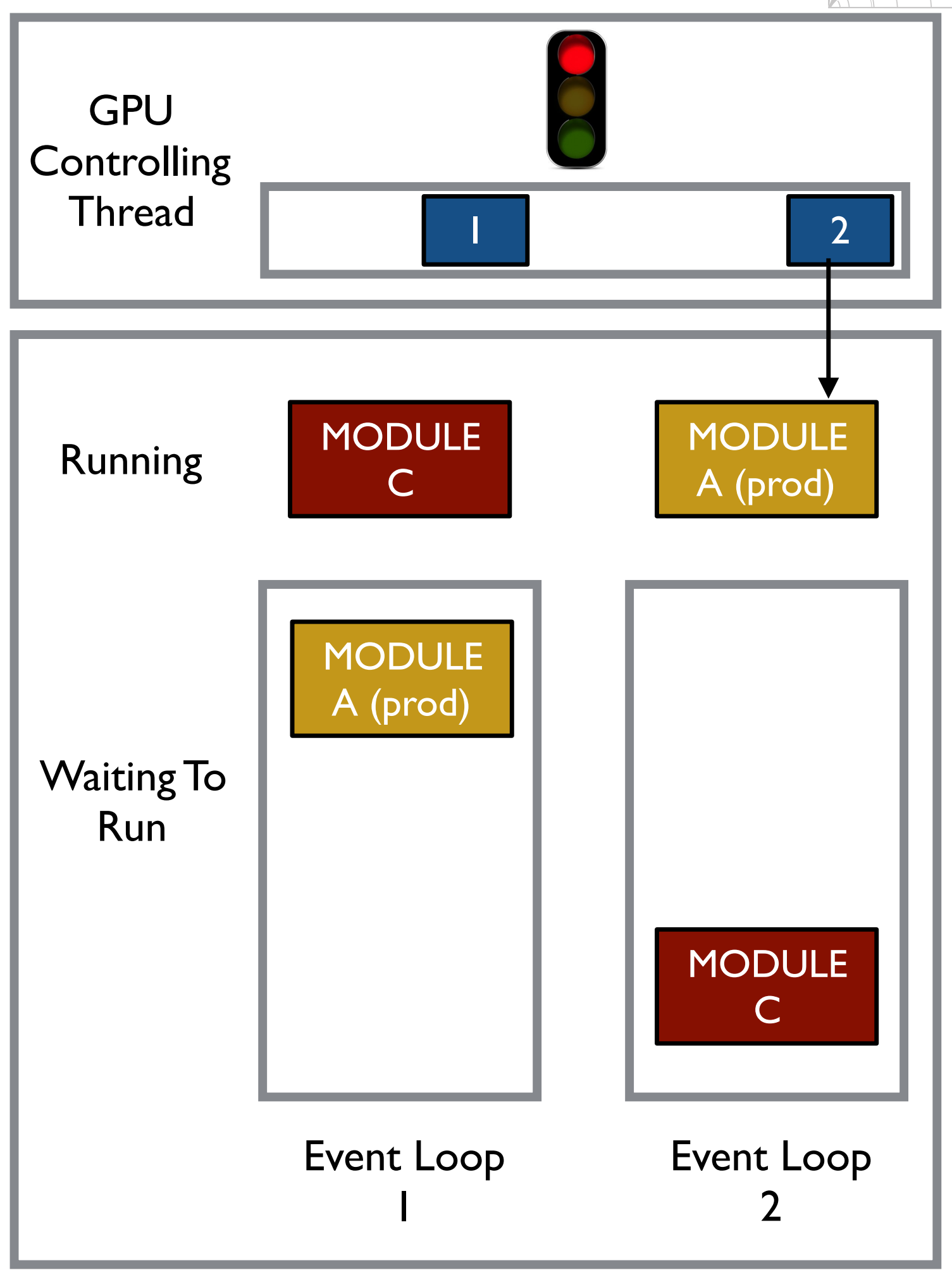

芼 Fermilab 


\section{External Work in Practice}

\section{GPU}

HLT base R\&D have been using the facility

See Matti Kortelainen's talk in this session

\section{Remote FPGA}

$R \& D$ project which uses remote (on or off site) FPGA for machine learning inference See Kevin Pedro's talk Wednesday in 'Reconstruction and Software Triggers'

\section{Geant V CMS Integration R\&D}

Control of thread passed to Geant $\mathrm{V}$ when new Event is ready

- Geant $V$ is using TBB task to run its code

- This allows proper thread pool sharing with framework also using TBB tasks

When Geant $\mathrm{V}$ finishes an Event, control returns to framework

- The finish thread often different than the start thread

See Kevin Pedro's talk Thursday in 'Detector Simulation' 


\section{External Work and Event Batching}

Framework supports running more concurrent events than threads

Normally not a useful configuration

- increases memory use

- does not increase event throughput

External Work modules can process events in batches

module waits to run external algorithm once it has acquired a certain number of events Has been used in framework tests

Not being used by any R\&D projects

- complicates module book-keeping

Some simple tests showed batching can decrease event throughput Have to carefully balance

- per module speed improvements from batching

- possible lack of available tasks for CPU to run while accumulating a batch 


\section{Configuring Heterogeneous Jobs}




\section{Heterogeneous Configuration}

Want jobs for a workflow to run at any site

Want same configuration for all jobs in a workflow

Be agnostic to the kind of hardware being used for a given job

Hash of configuration already used by framework to segregate data from different workflows

Want to be able to keep CPU and Accelerator algorithms separate

No need to touch working code

The different hardware may want to group the work differently

- e.g. CPU might want to spread over 3 modules while GPU wants them combined to 1 Not precluding having CPU and Accelerator algorithm in same module either

Use provenance tracking to determine what technology was used

Framework tracks what data each module uses for each Event 


\section{SwitchProducer}

SwitchProducer added to configuration

Allows specifying multiple modules associated to same module label

At runtime picks one to use based on available technologies
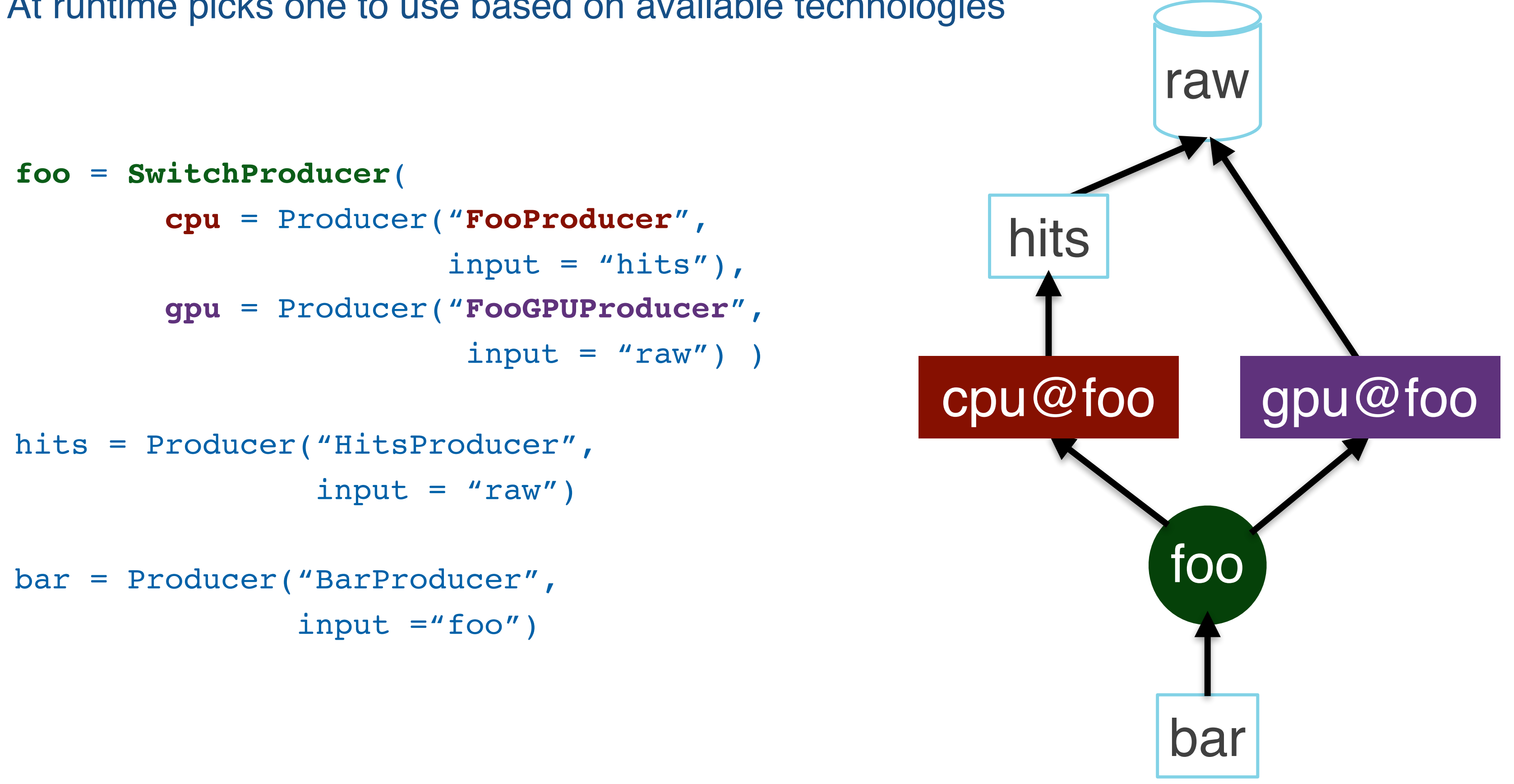


\section{Conclusion}

CMS has a mechanism for integrating TBB and accelerators

Can support any conceivable hardware

Possible future changes only after CMS gains more practical experience 


\section{Backup Slides}




\section{Throughput Scaling Test}

Approximate use of non-CPU resource

Separate helper thread which sleeps for a set amount of time All waiting sleep requests handled concurrently

- thread sleeps only for the longest requested time, not the total requested time Once sleeping, additional sleep requests will have to wait Denoted by 'External Work'

Simple CPU based algorithm for testing

algorithm sleeps for set amount of time

Require that two algorithms are needed to process each event

Test two different algorithm dependencies

The two algorithms are independent of each other

One algorithm depends on the results of the other algorithm 


\section{Expectations for Independent Algorithms}

\# threads = \# concurrent events both CPU algorithms take same time

\# threads = \# concurrent events

1 algorithm is faster than other

\# threads $=2{ }^{*} \#$ concurrent events both CPU algorithms take same time

\# threads = \# concurrent events

1 CPU \& 1 External Work algorithm
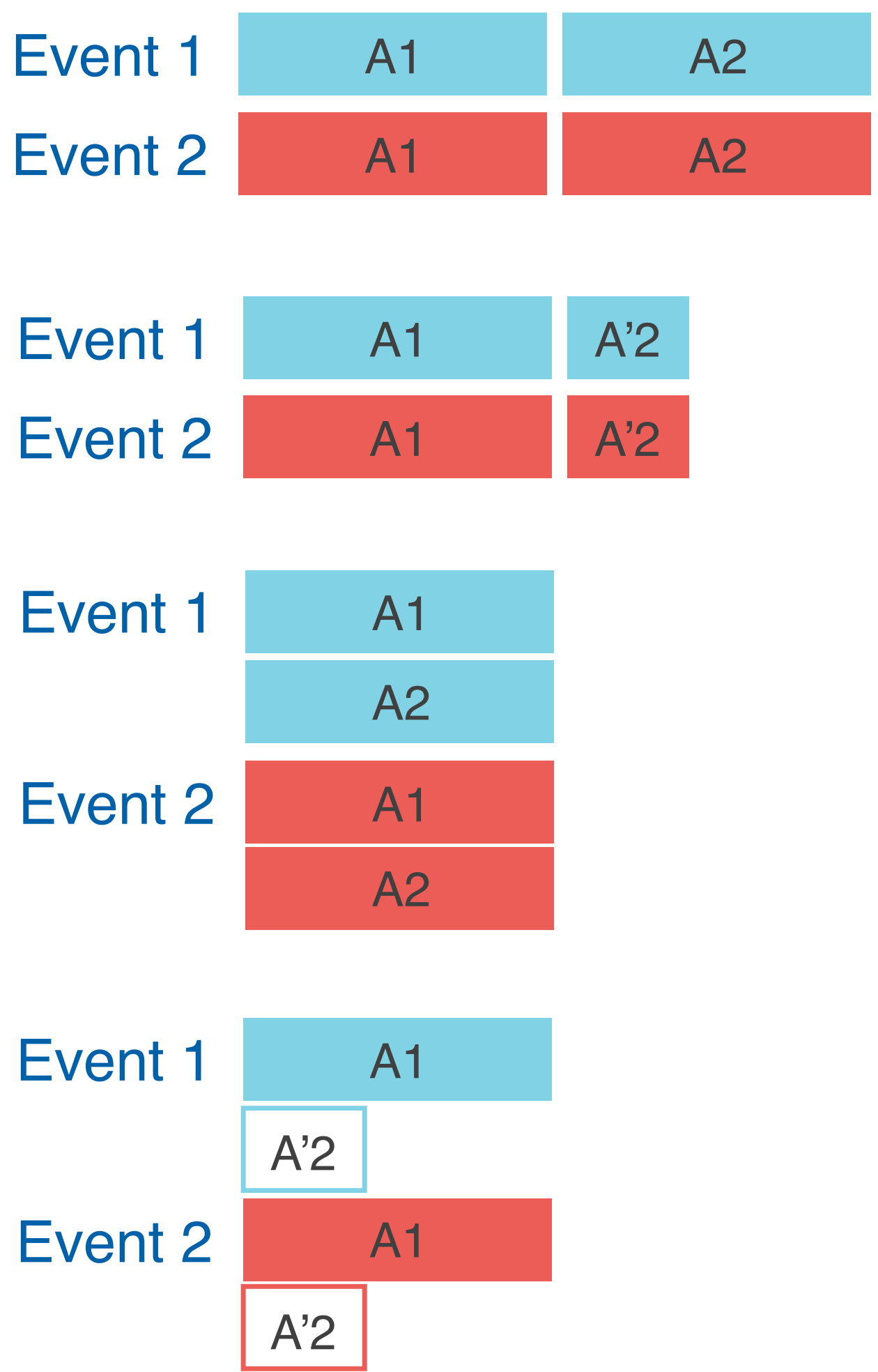


\section{Independent Algorithm Measurements}

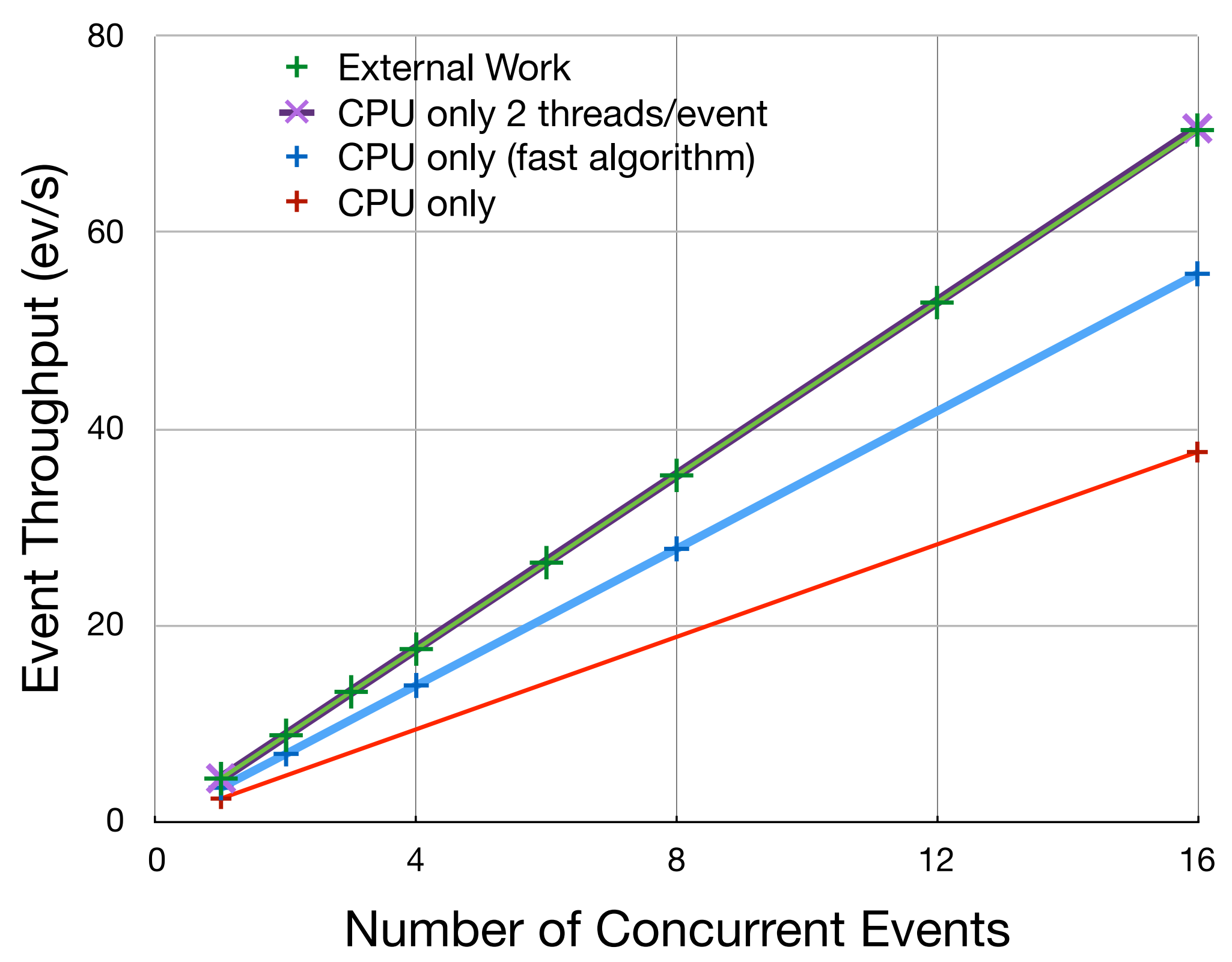

Have two algorithms that can work in parallel on one event

Algorithms take exactly the same amount of time to process an event

One algorithm can be written to do external work

As fast as using two threads per event 


\section{Processing Graph}

\section{Stream ID}

Denotes an independent event loop

\section{Histogram colors}

Purple: Work has been passed to the external work controlling thread

- Between acquire and produce

- Does not mean the work is running Green: a module is running on a CPU

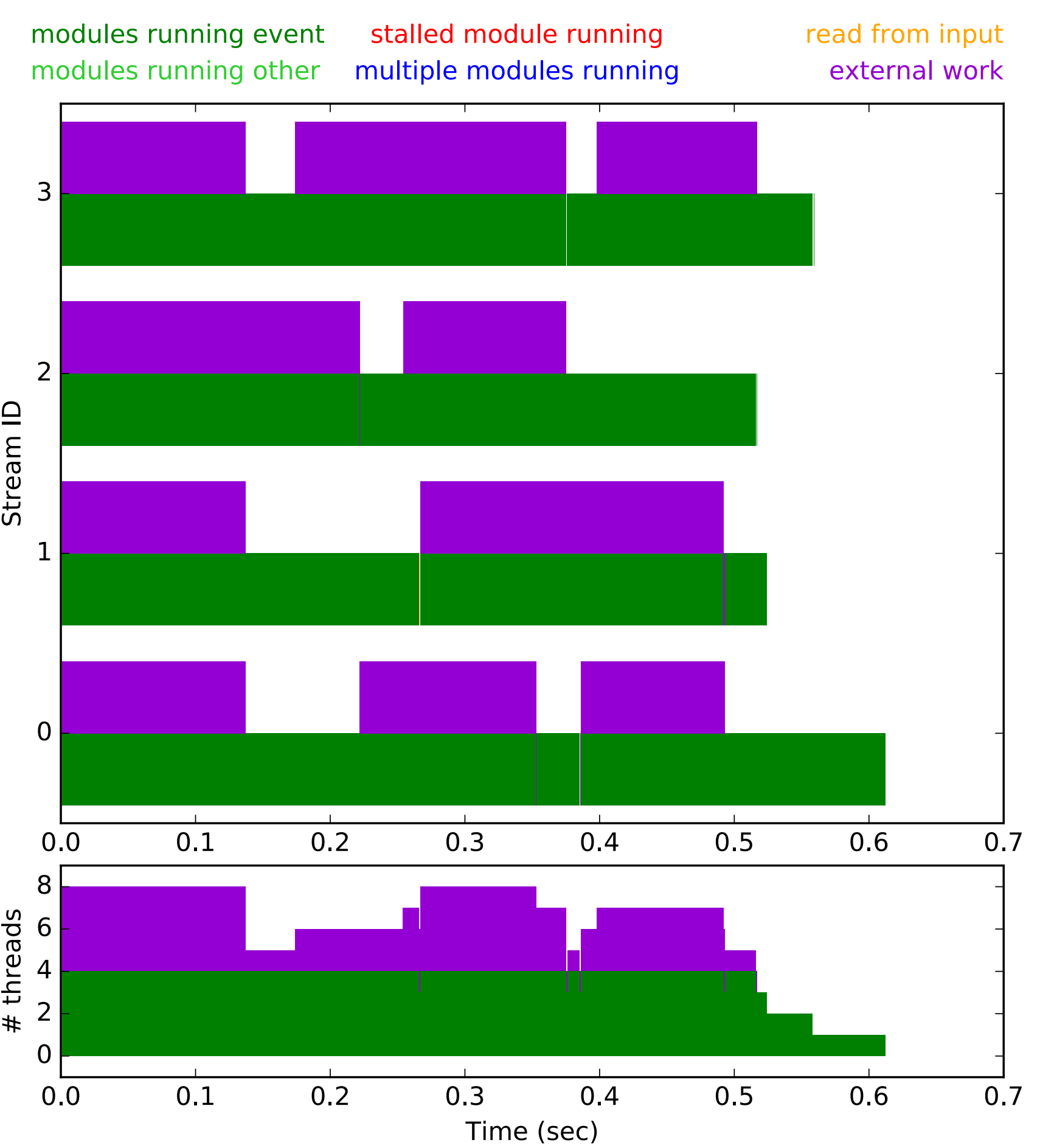




\section{Minimum Number of Events to Process}

The external work thread can wait until a set number of events are ready to process

Constants

16 concurrent events

16 threads

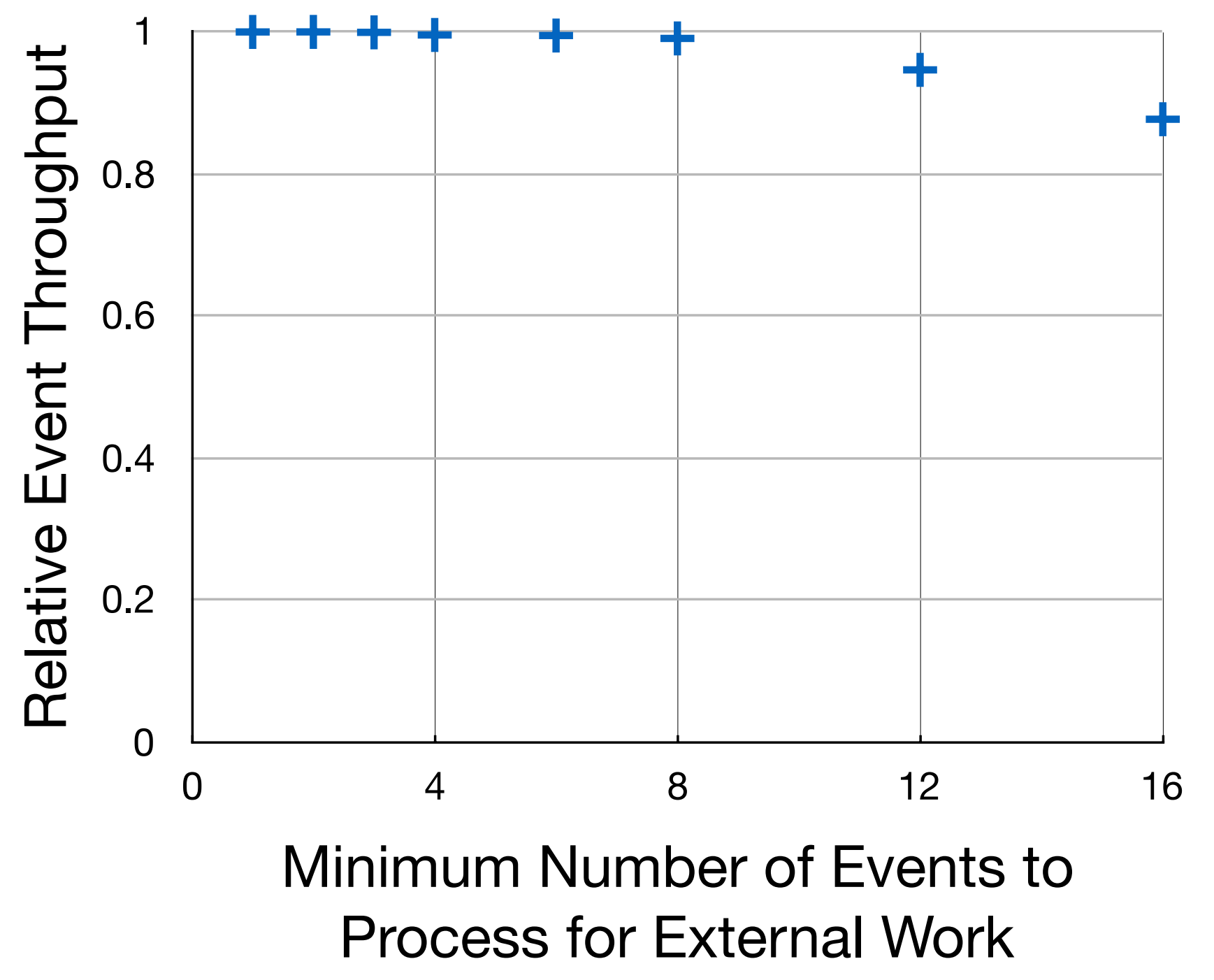

As minimum number of events approaches number of concurrent events the throughput decreases 


\section{Expectations for Dependent Algorithms}

\# threads = \# concurrent events

\begin{tabular}{l|l|l|} 
Event 1 & A1 & A2 \\
\hline Event 2 & A1 & A2 \\
\hline
\end{tabular}

\# threads = \# concurrent events

1 algorithm is faster than other

\# threads $=2{ }^{*} \#$ concurrent events

Event 1

\begin{tabular}{l|l}
$A 1$ & $A^{\prime} 2$ \\
$A 1$ & $A^{\prime} 2$
\end{tabular}

both CPU algorithms take same time No benefit from extra threads

Event 2

$A 2$

\# threads = \# concurrent events

1 CPU \& 1 External Work algorithm

Event 1

A1

A2

Event 2

A1
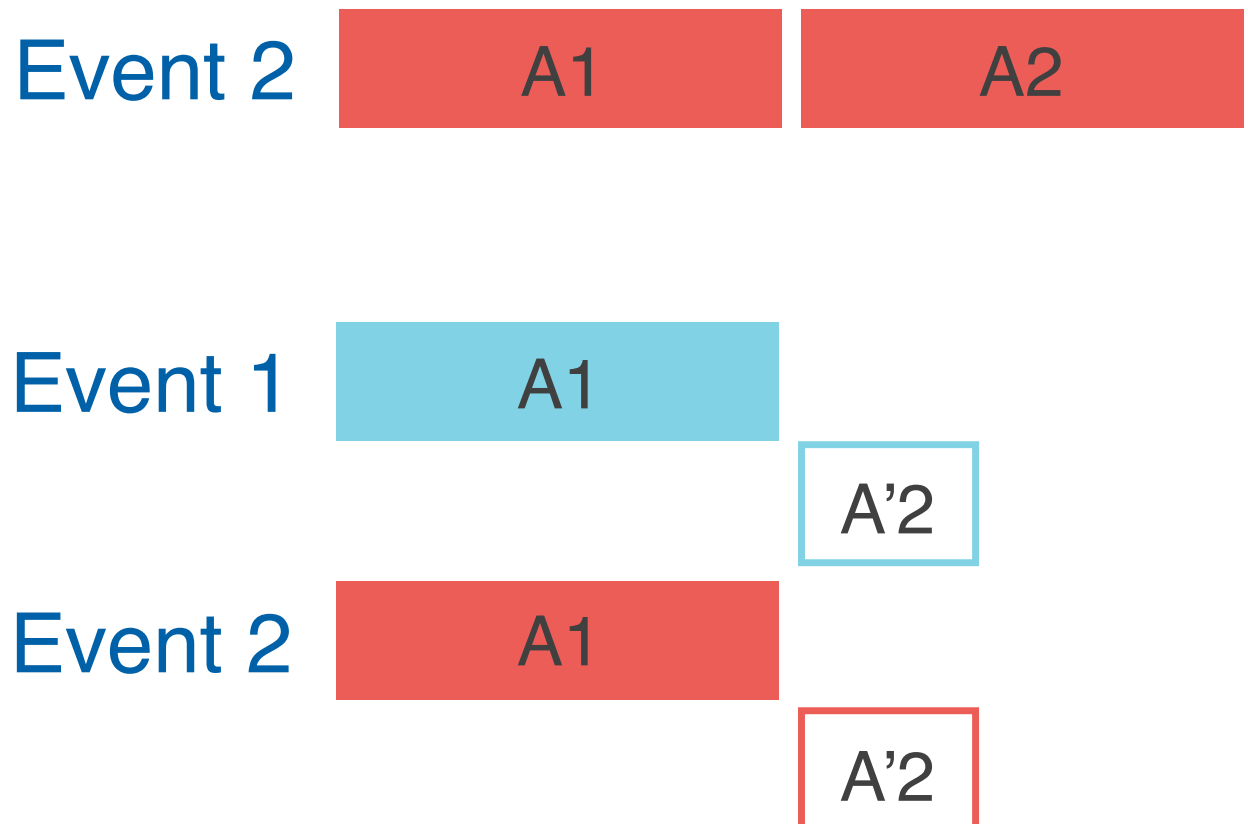


\section{Dependent Algorithm Measurements}

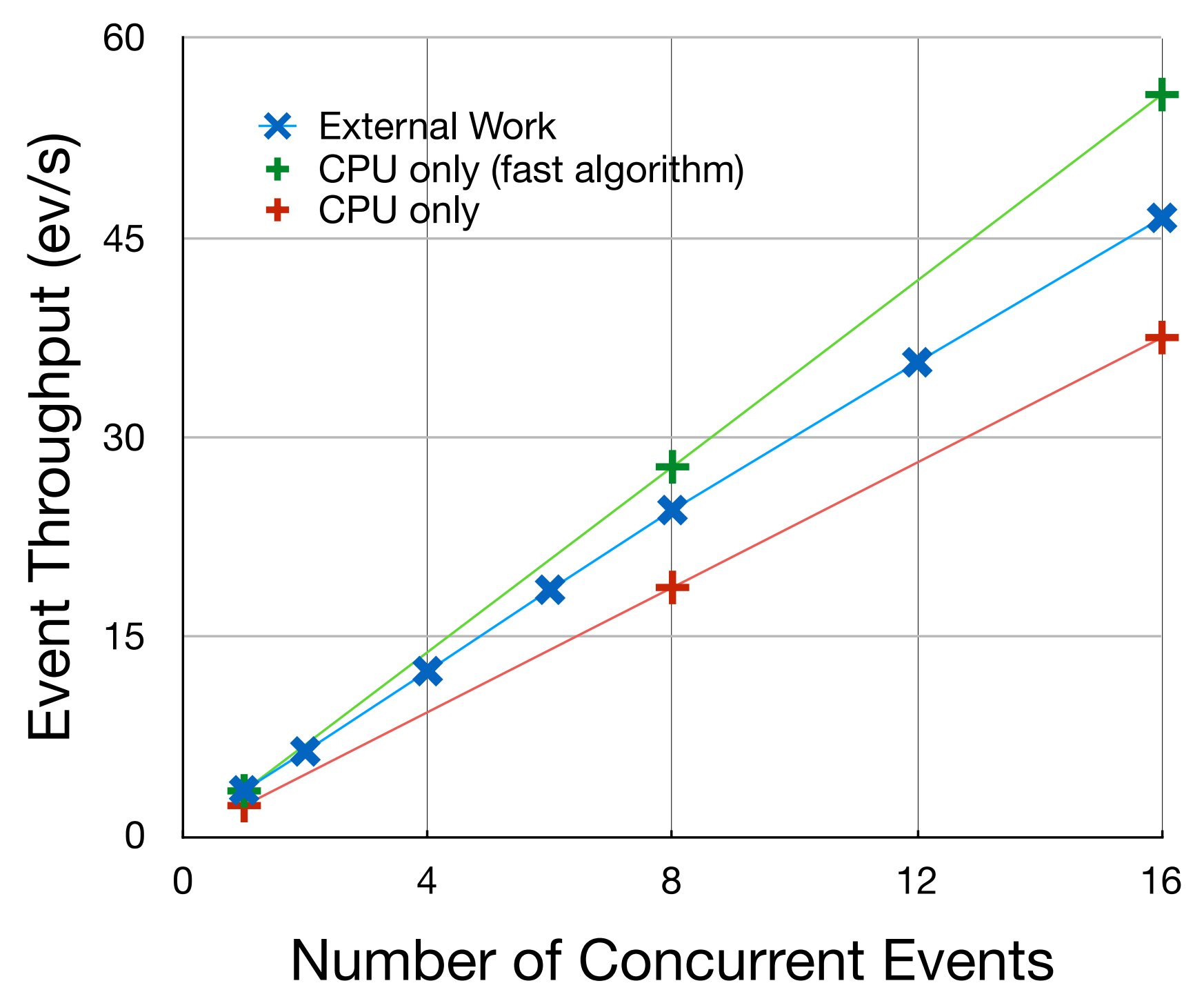

Event processing algorithms must run sequentially

Use of external work is faster than algorithms sequentially

not as fast as if second algorithm ran on CPU as fast as it can on external worker 


\section{Dependent Algorithm Processing Graphs}
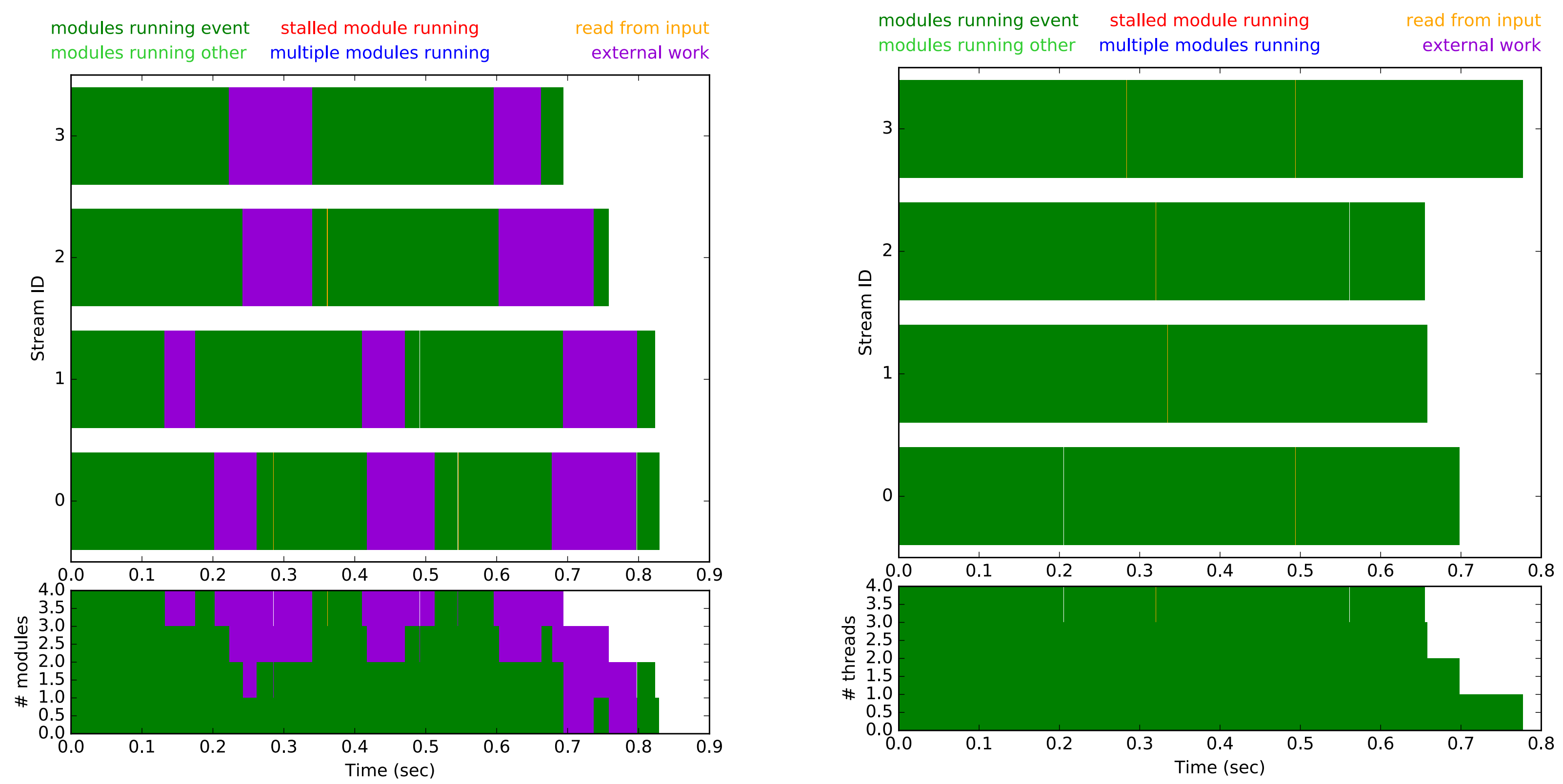

External Work and the CPU module have the same running times Note the scale change 


\title{
Cross Event Synchronization
}

\author{
External Worker
}

Event I

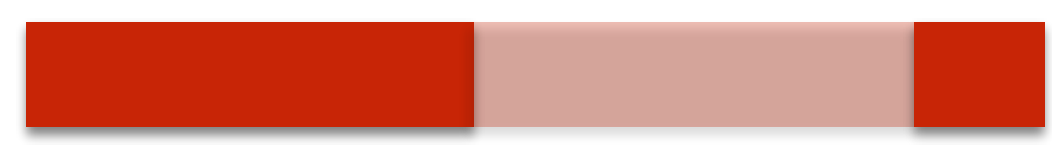

Event 2
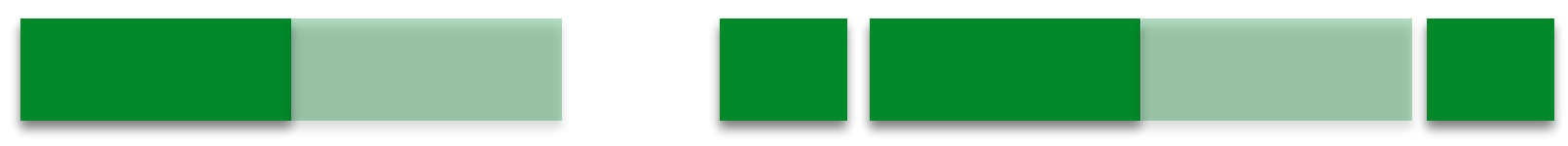

Event 3
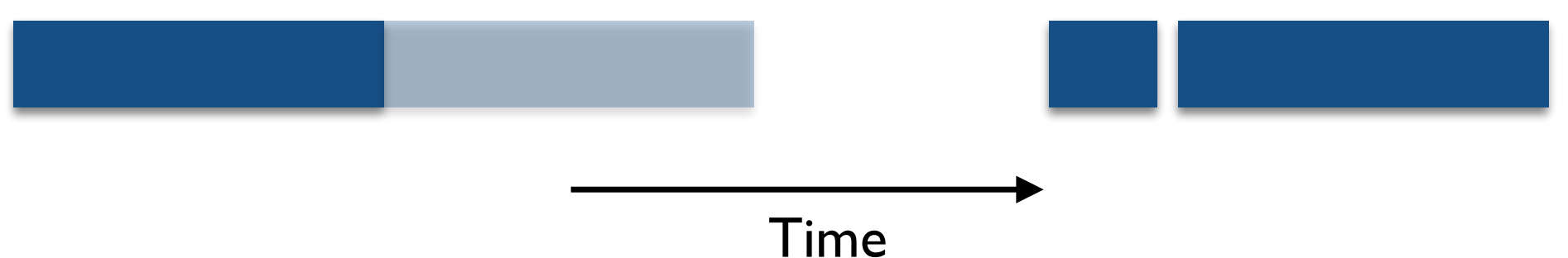

Key

Opaque:Time spent in algorithm/External worker

Semi transparent: amount of time to process data in the External Worker

Can only process 1 work chunk at a time

an event must wait for its turn if it missed the most recent start of a chunk

e.g. See Event 3

External work busy for the longest event time

events with shorter processing time must still wait for the longer time

e.g. see Event 2 


\section{Number of Concurrent Events $>$ Number of Threads}

Use 16 threads

Require external work to only wait for 1 event before processing

With enough concurrent events, can get same result as if the external work module was not in the job
Event Throughput vs Concurrent Events for External Work with 16 Threads

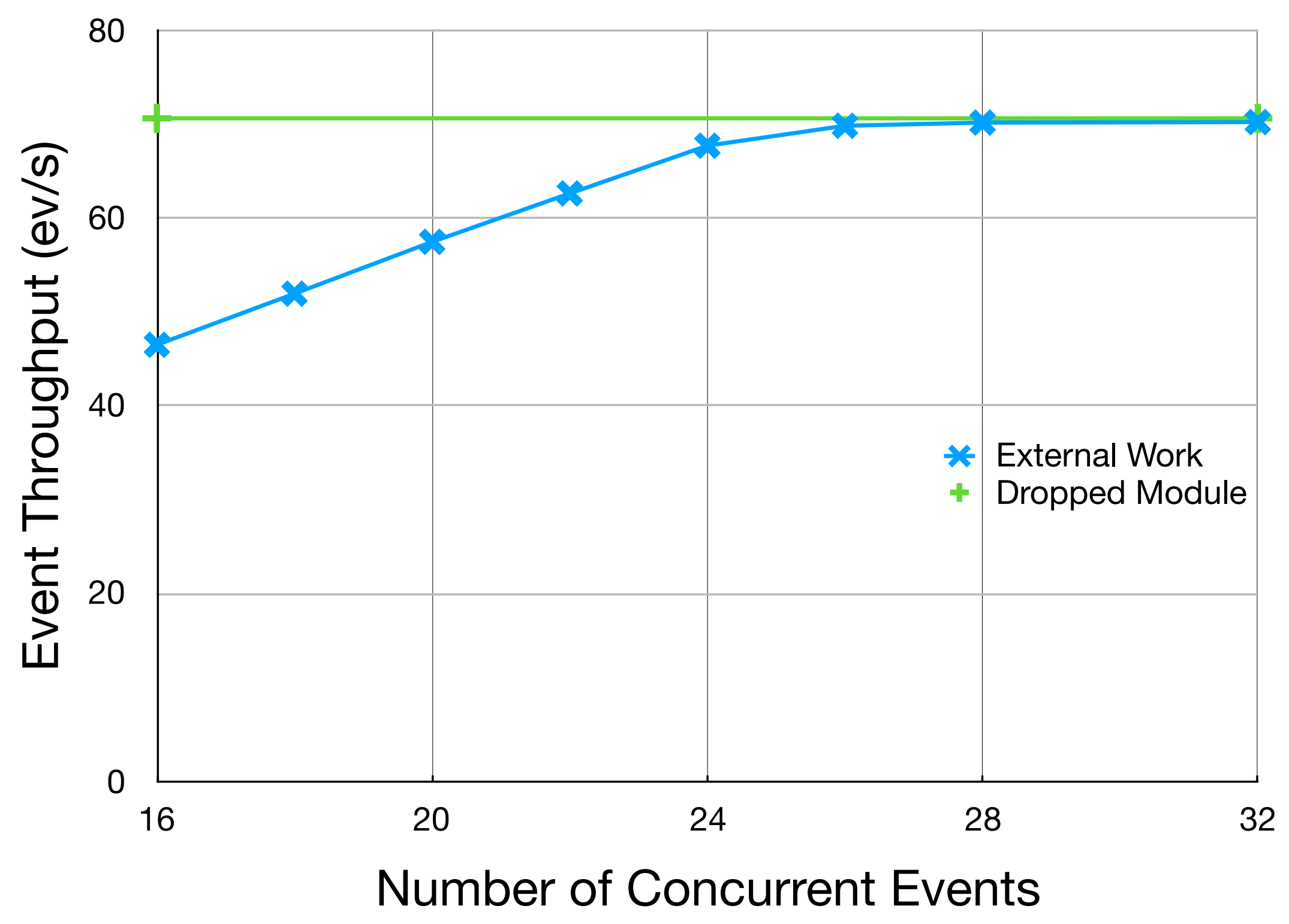




\section{Conclusion}

CMS has a mechanism for integrating TBB and accelerators

Exact event throughput benefits dependent on scheduling work to accelerator Waiting for enough events to accumulate can decrease throughput

The more intra-event parallelism improves event throughput

Can schedule work on CPU and accelerator at the same time

May be able to increase event throughput at the cost of extra memory

allow number of concurrent events to be greater than the number of CPU threads 